\title{
MODEL ORDER REDUCTION METHODS FOR COUPLED MACHINE TOOL MODELS
}

\author{
J. Vettermann ${ }^{1 *}$, S. Sauerzapf ${ }^{2}$, A. Naumann ${ }^{1}$, M. Beitelschmidt ${ }^{2}$, R. Herzog ${ }^{1}$, P. Benner ${ }^{1,3}$, J. Saak ${ }^{1,3}$ \\ ${ }^{1}$ TU Chemnitz, Faculty of Mathematics, Chemnitz, Germany \\ ${ }^{2} T U$ Dresden, Institute of Solid Mechanics, Chair of Dynamics and Mechanism Design, Dresden, Germany \\ ${ }^{3}$ Max Planck Institute for Dynamics of Complex Technical Systems, Magdeburg, Germany \\ ${ }^{*}$ Corresponding author; e-mail: julia.vettermann@mathematik.tu-chemnitz.de
}

\begin{abstract}
Thermal effects are the most dominant source for displacements in machine tools and thus workpiece inaccuracies during the manufacturing process. A promising strategy to meet the ever-increasing accuracy requirements is the use of predictive models for, e.g., parameter and design optimizations or online correction of the thermally induced error at the tool center point (TCP) in the production process. However, these techniques require fast but precise simulations. The need for high model accuracy is in direct contrast to the desired real-time capabilities. Model order reduction (MOR) is an attractive tool to overcome this problem. A modeling toolchain, which is tailored for the effective construction of fast and accurate models is proposed and demonstrated, emphasizing the involved MOR step.
\end{abstract}

\section{Keywords:}

model order reduction (MOR); coupled machine tool model; finite element discretization; simulation; thermo-elastic field

\section{INTRODUCTION}

Model order reduction is a successful and mathematically well understood approach to overcome the runtime restriction of high-dimensional simulation models. The resulting low-dimensional models enable fast simulations while preserving the model accuracy. Due to often drastically reduced memory requirements, an implementation directly into the numerical control system of machine tools becomes a possibility. Real-time applications, like correction of the thermally induced TCP errors [Ihlenfeldt 2019] and online parameter estimation [Herzog 2016], come into consideration.

The proposed modeling toolchain, which allows for the construction of detailed models in standard engineering software, aims at high flexibility with respect to the applied tools and numerical methods. Consequently, we allow for several newly developed simulation methods as well as geometric and physical coupling approaches, which have strong effects on the structure of the state-space systems used for simulations.

The efficiency of the new toolchain is shown for a thermal model of a demonstrator machine. In particular, several MOR methods are compared with regard to runtime and reduced model order. The advantages of these techniques are pointed out and concluding recommendations for different use cases are given.

The structure of the paper is shown in Fig. 1. Two coupling approaches, and therefore two structurally different input- output (IO) models, will be introduced in Section 2. Afterwards, both models will be reduced by MOR methods, specified in Section 3. Finally the reduced models will be compared in Section 4.

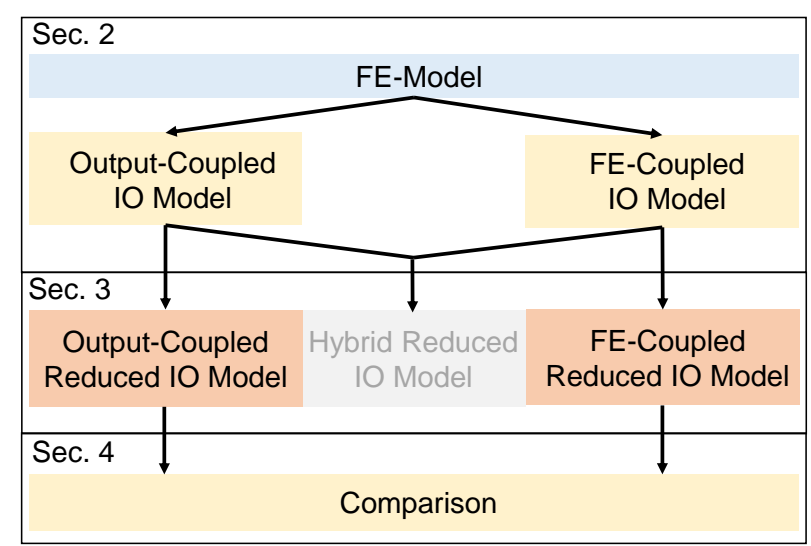

Fig. 1 : Structure of the paper divided into sections by model size and coupling approaches

\section{MACHINE TOOL MODEL}

\subsection{FE Model Generation}

A sufficiently detailed CAD model of the physical object under investigation is the basis of all modeling steps. After ap- 
plying necessary procedures to generate an efficient simulation model, such as removal of unnecessary geometric details, or filling cavities, the geometry model is used to define a finite element model (FE model). For the FE model definition in this publication, ANSYS Mechanical is used.

First, the geometry has to be structured into so called assemblies, which can move relative to each other. Each assembly can consist of several subassemblies. Since every subassembly represents a single finite element mesh in an assembly, we also refer to them as parts. Every part itself can consist of multiple bodies. Material properties are assigned to every single body, i.e., one body consists of a single material. Notice that this approach is restricted to piecewise constant material parameters like thermal conductivity and specific heat capacity.

Boundary conditions, like fluxes are applied to the outer surfaces of the parts. Couplings of the thermal fields of different parts can only exist between the outer surfaces of two parts and assemblies, respectively.

To calculate the thermal field, the heat equation

$c \rho \frac{\partial T(t)}{\partial t}-\vec{\nabla} \cdot(\lambda \vec{\nabla} T(t))=q(t)$

has to be discretized in space using finite elements (FE) and approximated with a suitable time step integrator.

The material coefficients are the specific heat $c$, the density $\rho$ and the thermal conductivity $\lambda$. The variable $T$ denotes the temperature at any given material point, $q$ represents a heat source and the variable $t$ signifies time. The application of the finite element method to equation (1) leads to the following system equation to calculate the discrete temperature vector $\boldsymbol{T}$ at the finite element nodes,

$\boldsymbol{E} \dot{\boldsymbol{T}}(t)=\boldsymbol{D} \boldsymbol{T}(t)+\boldsymbol{q}(t)$,

with matrices $\boldsymbol{E}$ (capacity matrix), $\boldsymbol{D}$ ((negative) conductivity matrix) and the time-dependent load vector $\boldsymbol{q}$. Moreover, $\dot{\boldsymbol{T}}$ denotes the time derivative of the temperature $\boldsymbol{T}$.

The Application Customization Toolkit (ACT) extension for ANSYS Mechanical allows to extend ANSYS with custom tools. We implemented the new toolchain with the ACT extension to define the model and generate a machine readable description of the continuous, coupled problem. This description is then fed into further tools to fulfill the tasks required, like generation of input-output (IO) models or simulation of the thermal field by a transient FE model simulation.

\subsection{From FE to IO Model}

The state-space system (2) hides the coupling between the temperature fields in the conductivity matrix $\boldsymbol{D}$ and the load vector $\boldsymbol{q}$. Furthermore, the capacity and conductivity matrices have block structure, where every block represents one part or, in other words, one subassembly.

We wish to analyze the effect of different coupling strategies on the efficiency of MOR methods. Hence we extend the standard state space system (1) to the block input-output system

$$
\begin{aligned}
\boldsymbol{E}_{i} \dot{\boldsymbol{T}}_{i} & =\left(\boldsymbol{D}_{i}+\boldsymbol{N}_{i}\right) \boldsymbol{T}_{i}+\boldsymbol{M}_{i j} \boldsymbol{T}_{j}+\boldsymbol{B}_{i} \boldsymbol{u}_{i}(t)+\boldsymbol{B}_{i, y} \boldsymbol{u}_{i, y}(y), \\
\boldsymbol{y}_{i} & =\boldsymbol{C}_{i} \boldsymbol{T}_{i},
\end{aligned}
$$

where the vector $\boldsymbol{T}_{\boldsymbol{i}}$ represents the temperature field of the i-th subassembly. Most terms in this equation are quite standard. As before, the matrices $\boldsymbol{E}_{i}$ and $\boldsymbol{D}_{i}$ represent the block capacity and the block conductivity matrices, respectively.
The input matrix $\boldsymbol{B}_{i}$ collects all inputs which do not depend on the solution temperature. These are the Neumann-type boundary conditions. Additionally, these matrices also contain the spatial resolution of the external temperatures. For these columns, the inputs correspond to the external temperature, scaled with the heat transfer coefficients. This information will be used in the MOR Section 3.

The output matrix $\boldsymbol{C}_{\boldsymbol{i}}$ maps the state to the outputs, i.e. observations. These can consist of the temperature values in finite element degrees of freedom, average temperatures across sensor areas, or, as in our case, the temperature averages on coupling surfaces.

The most uncommon terms are the input matrix $\boldsymbol{B}_{i, y}$ and the coupling coefficient matrices $\boldsymbol{N}_{i}$ and $\boldsymbol{M}_{i j}$. These coefficients arise from the thermal flux coupling of the machine tools subassemblies. We will compare two coupling approaches and describe them in the following section.

Please note that we can simplify the system into the standard block LTI (linear time-invariant) system form

$$
\begin{aligned}
\boldsymbol{E} \dot{\boldsymbol{x}}(t) & =\boldsymbol{A} \boldsymbol{x}(t)+\boldsymbol{B} \boldsymbol{u}(t), \\
\boldsymbol{y}(t) & =\boldsymbol{C} \boldsymbol{x}(t),
\end{aligned}
$$

where the vector $\boldsymbol{x}$ represents the concatenation of all state vectors $\boldsymbol{T}_{i}$ and the matrices $\boldsymbol{E}, \boldsymbol{A}, \boldsymbol{B}$ and $\boldsymbol{C}$ consequently are block matrices. Notice further that the matrix $\boldsymbol{A}$ comprises the submatrices $\boldsymbol{D}_{i}$ and $\boldsymbol{N}_{i}$ on the diagonal blocks and the matrices $\boldsymbol{M}_{i j}$ in the off-diagonal blocks. The output dependency is hidden in the new vector-valued input functions $\boldsymbol{u}(t)$.

\subsection{Coupling Approaches}

The coupling in the coupled system of heat equations is realized by heat fluxes. The fluxes connect the temperature fields of the subassemblies. These fluxes in general depend on both temperature fields connected to the joint surface, which makes them time- and space-dependent. The temperature field of every subassembly is defined only on the corresponding geometry, and particularly on the corresponding mesh. Hence, these coupling fluxes require a coupling strategy.

To simplify the notation, we will consider only linear coupling fluxes of the form $\boldsymbol{q}_{j}=\alpha *\left(\boldsymbol{T}_{i}-\boldsymbol{T}_{j}\right)$ at a surface of the $\mathrm{j}$-th subassembly, where the heat transfer coefficient $\alpha$ is a constant.

\section{Output-Coupled IO Model}

The finite element model already contains outputs, which represent quantities of interest. Therefore, the simplest idea is to compute the average temperature at the coupling surface and use that as an output. Furthermore we split the flux into $\alpha \boldsymbol{T}_{i}$ and $-\alpha \boldsymbol{T}_{j}$. The second term leads to the standard surface mass matrix and constitutes the matrix $\boldsymbol{N}_{i}$. The first term will use the corresponding output $y_{i}$ together with an input vector for the input matrix $\boldsymbol{B}_{i, y}$.

We refer to this system, which uses the averaged surface temperature for the coupling, as the output-coupled system. Due to the output-coupling approach, we have at least as many outputs as there are coupling boundary conditions.

\section{FE-Coupled IO Model}

Our second coupling strategy uses the finite element approximation of the temperature fields and the mortar approach as implemented in [Bastian 2009].

Instead of approximating the temperature at the coupling surface, we treat one component as a master and the other one as the slave. Then we project the slave mesh onto the master mesh, together with the temperature field. This 
leads to a common mesh, on which we compute the coupling matrices $\boldsymbol{N}_{i}$ and $\boldsymbol{M}_{i j}$.

We call the system with the FE-coupled coefficient matrices $\boldsymbol{M}$ and $\boldsymbol{N}$ the FE-coupled system. Keep in mind that in this case we have only the desired outputs from the users, no additional ones. Please note, that we use the mortar approach only as a coupling approach between the temperature fields.

Both approaches lead to differently structured LTI systems The output-coupled system is block diagonal, since the coupling terms are hidden in the inputs. It is this block diagonal structure, which gives rise to a serial time integration approach, where each block is solved at a time.

By contrast, the FE-coupled system is a full block system, where the off-diagonal blocks stem from the coupling matrices. That system is not solvable in a serial way and all systems have to be integrated in time at once.

Furthermore, the heat equation belongs to the set of standard examples of stiff ordinary differential equations (ODE) see the third example in [Hairer 2010, Ch. IV]. And thus an implicit integrator has to be used. In turn, the diagonal block system looks advantageous. The advantage vanishes in case the coupling terms dominate. In that case, one has to reduce the time step size, or incorporate the coupling terms into the Jacobian matrix to retain stability.

\section{MOR FOR COUPLED MODELS}

During the process of model order reduction, an approximation of a high-dimensional simulation model is computed. In direct consequence, this low-dimensional reduced order model (ROM) is used as a surrogate model in applications where either fast model evaluations are required or storage capacity is limited. Thereby, the essential information of the dynamic behavior of the original system is preserved in the ROM, while redundancy is eliminated and so its matrix dimension is drastically reduced. For a general overview on MOR techniques for coupled problems see [Benner 2015a]. Here, we focus on those aspects relevant for the purpose of simulating machine tools. The value of MOR in this field is also presented in [Hernandes-Becerro 2020].

\subsection{Basic Principle}

As foundation of the system-theoretic MOR techniques used here, see e.g. [Antoulas 2005], [Baur 2014], an inputoutput model (4) in generalized state space form is considered. The goal of projection based MOR is the identification of truncation matrices $\boldsymbol{V}, \boldsymbol{W} \in \mathbb{R}^{n \times r}$ to restrict the model to a low-dimensional subspace. The specific determination of $\boldsymbol{V}$ and $\boldsymbol{W}$ is dependent on the MOR method in use. The reduced system of order $r \ll n$ is then obtained by

$$
\begin{aligned}
\boldsymbol{W}^{T} \boldsymbol{E} \boldsymbol{V} \dot{\boldsymbol{x}}_{r}(t) & =\boldsymbol{W}^{T} \boldsymbol{A} \boldsymbol{V} \boldsymbol{x}_{r}(t)+\boldsymbol{W}^{T} \boldsymbol{B} \boldsymbol{u}(t), \\
\boldsymbol{y}_{r}(t) & =\boldsymbol{C} \boldsymbol{V} \boldsymbol{x}_{r}(t),
\end{aligned}
$$

with the reduced state $\boldsymbol{x}_{r} \in \mathbb{R}^{r}$ and the output $\boldsymbol{y}_{r} \approx \boldsymbol{y} \in \mathbb{R}^{p}$ of the ROM. We require that the output error $\left\|\boldsymbol{y}(t)-\boldsymbol{y}_{r}(t)\right\|$ is small in a suitable norm and emphasize that the focus of our MOR approach lies on finding a good approximation of the model output $\boldsymbol{y}(t)$. In turn, it does not necessarily provide an accurate approximation $\widehat{\boldsymbol{x}}(t)=\boldsymbol{V} \boldsymbol{x}_{r}(t)$ for the entire high dimensional state $\boldsymbol{x}(t)$.

The transfer function $\boldsymbol{H}(s) \in \mathbb{C}^{p \times m}$ with
$\boldsymbol{H}(s)=\boldsymbol{C}(s \boldsymbol{E}-\boldsymbol{A})^{-\mathbf{1}} \boldsymbol{B}$

describes the relation between the output response $\boldsymbol{Y}(s)=$ $\boldsymbol{H}(s) \boldsymbol{U}(\mathrm{s})$ and the input signal $\boldsymbol{U}(s)$ of the system in frequency domain, while assuming a zero initial state $x(0)=$ $x_{0}=0$. The representation in (6) is obtained by applying the Laplace transformation to (4) [Antoulas 2005, Ch. 5]. In the considered system theoretic MOR approach, the input-output behavior of the reduced system is adjusted to that of the original model by an approximation of the transfer function (6).

A second class of MOR methods should be mentioned at this point. In the simulation based MOR approach, repeated model evaluations, so called snap shots, are needed to establish the projection matrices. Well known training based techniques are, e.g. the Reduced Basis method and Proper Orthogonal Decomposition (POD), see e.g. Chapters 1-3 in [Benner 2017]. But these approaches show less flexibility towards changing load cases, compared to those used during the reduction process, while the system theoretic MOR methods work independently of the concrete input $\boldsymbol{u}$.

Additionally, modal analysis is a widely available option in FE tools. While being rather successful in many mechanical applications, this approximation type is usually not a competitive option for thermal problems, e.g. [Benner 2006].

In the following, a short description of two commonly used system theoretic MOR methods and their advantages is given. They differ in the way the truncation matrices $\boldsymbol{V}$ and $\boldsymbol{W}$ are determined.

Energy-Based Reduction via Balanced Truncation (BT)

As its name implies, this method seeks to find a balanced realization of the system that allows the identification and truncation of states which are of little importance for the input-output behavior of the underlying dynamical system. Balanced truncation is a popular reduction method due to some desirable features: first, the stability of the original model is preserved in the ROM and second, the method provides an a priori error bound, which can be used to determine the required reduced order for a given tolerance on the approximation error, see (9).

The system Gramians, see e.g. [Antoulas 2005, Ch.4], play an essential role in BT. They are related to the energy of the system: the smallest amount of energy needed to reach the state $\overline{\boldsymbol{x}}$ from a zero initial state is given by $\overline{\boldsymbol{x}} \boldsymbol{P}^{-1} \overline{\boldsymbol{x}}$ and the expression $\overline{\boldsymbol{x}}^{T} \boldsymbol{E}^{T} \boldsymbol{Q} \boldsymbol{E} \overline{\boldsymbol{x}}$ denotes the output energy observed in a system with initial condition $\bar{x}$ and no excitation. The states which require a large amount of energy to be reached and/or obtain a small amount of observation energy can be eliminated from the system. However, this concept depends on the basis and therefore a basis in which these two concepts coincide is required. This is fulfilled in a balanced basis. Then the Gramians are equal and diagonal, and moreover, states which are hard to reach are simultaneously hard to observe.

The reachability Gramian $\boldsymbol{P}$ and the observability Gramian $\boldsymbol{E}^{T} \boldsymbol{Q E}$ can be determined using the following equalities

$$
\begin{aligned}
& \boldsymbol{A P} \boldsymbol{E}^{T}+\boldsymbol{E P} \boldsymbol{A}^{T}+\boldsymbol{B} \boldsymbol{B}^{T}=0, \\
& \boldsymbol{A}^{T} \boldsymbol{Q E}+\boldsymbol{E}^{T} \boldsymbol{Q A}+\boldsymbol{C} \boldsymbol{C}^{T}=0 .
\end{aligned}
$$

Then the states which shall be kept in the reduced model can be identified by the magnitude of the Hankel singular values (HSV) $\sigma_{i}$, which are the square roots of the eigenvalues $\lambda_{i}$ of the Gramians, see [Antoulas 2005, Ch. 7], given as 
$\boldsymbol{\sigma}_{i}=\sqrt{\lambda_{i}\left(\boldsymbol{P} \boldsymbol{E}^{T} \boldsymbol{Q E}\right)}, \quad i=1, \ldots, n$.

Note that for a balanced system realization, the HSV coincide with the diagonal entries of the Gramians. It can be shown that the maximum of the frequency response $\left(\mathcal{H}_{\infty}-\right.$ norm) of the error between the transfer functions of the full and the reduced order models is bounded above by twice the sum of the neglected HSVs

$\left\|\boldsymbol{H}-\boldsymbol{H}_{r}\right\|_{\mathcal{H}_{\infty}} \leq 2 \sum_{k=r+1}^{n} \boldsymbol{\sigma}_{k}$.

An expression for the output error between the full and the $\mathrm{ROM}$ is obtained by

$\left\|\boldsymbol{y}-\boldsymbol{y}_{\boldsymbol{r}}\right\|_{\mathcal{L}_{2}} \leq\left\|\boldsymbol{H}-\boldsymbol{H}_{r}\right\|_{\mathcal{H}_{\infty}}\|\boldsymbol{u}\|_{\mathcal{L}_{2}}$

see, e.g. [Baur 2014]. Hence, we recommend to design the system such that the entries in the input $\boldsymbol{u}$ stay moderately sized, i.e. not much bigger than one. For example, large constant heat conductivity coefficients should be included in the input matrix $\boldsymbol{B}$. A scaling of the matrix $\boldsymbol{B}$ according to the magnitude of the corresponding inputs for the reduction process is also an option for handling this instance.

This reduction method is implemented for large sparse systems in the open source MATLAB $®$ tool M-M.E.S.S. - the MATLAB Matrix Equation Sparse Solvers [Benner 2020].

Moment Matching and Interpolation of the Transfer Function

The goal of basic moment matching methods is the computation of a ROM whose transfer function interpolates that of the original model in some given complex interpolation points. More specifically, some of the coefficients of the Taylor expansion, the so called moments, at one or several expansion points $s_{i} \in \mathbb{C}, i=1, \ldots k$; of the transfer functions $\boldsymbol{H}$ and $\boldsymbol{H}_{\boldsymbol{r}}$ of the full and the reduced systems are matched. There are different variations of moment matching, which differ in the choice of the number and the location of the expansion points, as well as the number of moments to match. These MOR methods are based on Krylov subspaces, which are typically computed via the Arnoldi and Lanczos algorithms (see, e.g. [Antoulas 2005]).

A very effective algorithm, the Iterative Rational Krylov Algorithm (IRKA), introduced in [Antoulas 2008] provides two valuable properties. The reduced transfer function $\boldsymbol{H}_{\boldsymbol{r}}(s)$ is a locally optimal approximation of $\boldsymbol{H}(s)$ with respect to the $\mathcal{H}_{2}$ norm among all reduced order systems having the same reduced order. IRKA iteratively and automatically adapts the expansion points within the reduction procedure and thus is easy to apply and suited for semi-automatic MOR. This algorithm is also implemented in M-M.E.S.S.

In contrast to BT, there is no unconditional guarantee for the preservation of stability in the reduced system for the interpolation-based MOR approaches. Furthermore, for these MOR techniques no cheap to evaluate a priori error bound exists, which would allow for an easy adaptive computation of the reduced order $r$. However a posteriori error bounds exist and the $\mathcal{H}_{2}$ error can be computed with reasonable effort.

\section{Parametric MOR}

To limit the size of this paper we restrict our considerations of MOR for coupled models to LTI systems. Nevertheless, there are several techniques to handle models with parameter dependent system matrices in the field of MOR, see e.g. [Benner 2015b], which can easily be combined with the methods for coupled models outlined in the next section. They enable the preservation of physical parameters, such as material-dependent heat conductivity coefficients, in the ROM.

\subsection{MOR for FE- and Output-Coupled Models}

The two coupling approaches outlined above have direct consequences on the reduction process and the dimension as well as the structure of the computed ROMs. In the following, the advantages and disadvantages of FE- and output-coupled models are discussed with regard to MOR.

\section{Output-Coupled Model}

As the name already indicates, the coupling is established by contact information in the inputs and outputs of the individual subassemblies. This approach has the advantage that the subassemblies can be considered as single models with additional internal inputs and outputs and thus can be reduced separately. Therefore, the ROM has the same network structure as the full order model and single subassembly models can be replaced separately, without the need to recompute the rest. Just the inputs and outputs of this substituted part have to coincide with that of the original subassembly for proper contact information within the overall model. Furthermore, the method and parameters for the reduction process can be adapted to the actual requirements of every single subassembly. So, this approach proves to be very flexible. Also faster offline computation of the ROMs for the low-dimensional single subassemblies with dimensions $n_{j}, j=1, \ldots, k$; compared with the reduction time for the whole system of dimension $n=\sum_{j=1}^{k} n_{j}$, is achievable.

However, in our experience a high number of inputs and outputs between subassemblies also leads to higher reduced orders of the separate and, consequently, the overall model. Thus, higher online simulation times and memory requirements of the reduced system have to be expected. This circumstance will be explained in more detail in the next paragraph. Moreover, the reduction process cannot take advantage of the relative importance of the single subassemblies.

\section{FE-Coupled Model}

The coupling, which connects the subassemblies on the FE-level, is realized directly in the system matrix $\boldsymbol{A}$ by coupling blocks $\boldsymbol{M}_{\boldsymbol{i} \boldsymbol{j}}$, see (3). This results in the fact, that the subassemblies cannot be reduced separately anymore rather, the overall model of the machine tool has to be reduced as a whole. Consequently, the computational effort for the reduction process is high (offline) and the subassembly structure is lost in the ROM. In contrast to the outputcoupled models, the reduced subassemblies are not exchangeable. Therefore, a new reduction process of the entire machine is required for every modification in the model structure.

Due to the consideration of the internal inputs and outputs within the coupling blocks in $\boldsymbol{A}$, just the external ones are left as model inputs and outputs. So, the total number of inputs and outputs of the model is much smaller than in the output-coupled case. This proves to be advantageous for the process of MOR, because the dimensions $m$ of the input matrix $\boldsymbol{B}$ and $p$ of the output matrix $\boldsymbol{C}$ directly influence the computational costs and the achievable reduced order within a given tolerance. This follows from two facts: first, the repeated solution of linear systems of equations (LSE) is an essential part of the reduction methods and the dominant cost to solve the LSE is dependent on the number of model inputs and outputs. Second, the purpose of the system theoretic MOR techniques used here has to be considered: the input-output behavior of the dynamical system is approximated. So, the smaller the number of inputs and outputs, the fewer pathways between them need to be pre- 
served in the ROM and thus the order of the reduced system can be comparatively small. Consequently, less simulation time (online) as well as less memory capacity is needed for the FE-coupled ROM.

\subsection{Inhomogeneous Initial Conditions}

An often neglected point in the application of system theoretic MOR techniques is the fact that these methods rely on the restrictive assumption of a zero initial condition $x(0)=$ $x_{0}=0$. No transient system information, including the initial condition, is contained in the transfer function (6), which these MOR methods approximate. For instance, the common BT error bound (9) is assured only for systems with a homogeneous initial condition. The accuracy of the ROM, however, depends on a proper representation of the initial condition in the low-dimensional subspaces computed by the MOR routine.

The intuitive idea to rewrite the system (4) in terms of a state vector $\widetilde{\boldsymbol{x}}(t)=\boldsymbol{x}(t)-\boldsymbol{x}_{0}$ shifted by the nonzero initial condition $x_{0}$ for the reduction process, can be efficient for systems with a single a priori known initial value. However, various initial conditions may arise due to changes in the manufacturing process of a machine tool. At this point it is, obviously, economically not desirable to cool down the machine to the basic initial temperature at rest. If different initia conditions have to be considered, in a simulation with the ROM, other techniques should be employed. In the literature two leading methods to deal with this issue are suggested.

\section{Augmented System}

This technique is described in [Antoulas 2011] and is based on an extension of the system (4) by an augmented input including the information on the initial condition in the system to reduce. Following this idea, a matrix $\boldsymbol{E}_{\boldsymbol{0}}$ and a corresponding input $\boldsymbol{u}_{\mathbf{0}}(t)=\boldsymbol{z}_{\mathbf{0}} \delta(t)$, where $\delta(t)$ denotes the Dirac delta distribution, is added to (4). Hence, the system

$$
\begin{aligned}
\boldsymbol{E} \dot{\boldsymbol{x}}(t) & =\boldsymbol{A} \boldsymbol{x}(t)+\left[\begin{array}{ll}
\boldsymbol{B} \boldsymbol{X}_{0}
\end{array}\right]\left[\begin{array}{c}
\boldsymbol{u}(t) \\
\boldsymbol{u}_{0}(t)
\end{array}\right], \quad \boldsymbol{x}_{0}=0, \\
\boldsymbol{y}(t) & =\boldsymbol{C} \boldsymbol{x}(t)
\end{aligned}
$$

is used for the computation of the ROM. It is assumed that the matrix $\boldsymbol{X}_{0} \in \mathbb{R}^{\boldsymbol{n} \times \boldsymbol{n}_{\mathbf{0}}}$ spans a subspace $\mathbb{X} \subset \mathbb{R}^{n}$ which includes all feasible initial values such that $\boldsymbol{x}_{0}=\boldsymbol{X}_{0} \boldsymbol{z}_{0}$ with $z_{0} \in \mathbb{R}^{n_{0}}$. In the following, we call this approach BTX0.

\section{Additional System}

The idea of the augmented system is enhanced in [Beattie 2017]. To reach more adaptability in the reduction process the auxiliary input is shifted to a separate system

$\boldsymbol{E} \dot{\boldsymbol{w}}(t)=\boldsymbol{A w}(t)+\boldsymbol{E} \boldsymbol{X}_{0} \boldsymbol{u}_{0}(t), \quad \boldsymbol{w}(0)=\boldsymbol{w}_{0}=0$, $\boldsymbol{y}_{x_{0}}(t)=\boldsymbol{C} \boldsymbol{w}(t)$.

As result of this splitting into two models, the systems (4) and (12) can be reduced individually using appropriate MOR settings according to the particular model requirements. Due to the linearity of the systems the output $\boldsymbol{y}_{r}(t)$ of the reduced linear system (5) with initial condition $\boldsymbol{x}_{0} \neq 0$ is a superposition of the outputs of (5) with zero initial condition $x_{0}=0$ and (12) with input $\boldsymbol{u}_{0}(t)=\boldsymbol{z}_{0} \delta(t)$. This approach is more flexible, and shows better approximation properties at the cost of more effort for the ROM computation and simulation due to a second ROM required for an appropriate consideration of the influence of the initial condition to the dynamical behavior of the system. In our numerical investigations in Section 4, we refer to this approach as 2Phase.
A new variant of BT for systems with inhomogeneous initial conditions, which incorporates the two methods outlined above, can be found in [Schroeder 2020].

Notice that it is desirable to keep the dimension $n_{0}$ of $\boldsymbol{X}_{\mathbf{0}}$ small. Otherwise special MOR techniques for systems with many inputs, e.g. [Benner 2013], may be required, introducing an additional outer approximation step, and consequently additional approximation errors.

\section{THERMAL MODEL OF A DEMONSTRATOR MACHINE}

\subsection{Model Description}

An application of the toolchain for a simplified test machine can be found in [Beitelschmidt 2020].

To demonstrate the usage and benefits of the proposed methods for large, complex systems, a model of an experimental machine tool, called MAX, is used. The geometric model with all significant assemblies is shown in Fig. 2. Special characteristics of MAX are its light-weight construction and sledges consisting of aluminium plates, resulting in potentially large thermal deformations and a displacement of the workpiece relative to the spindle by the movement of the sledges.

After applying all necessary geometric simplifications, the finite element model, shown in Fig. 3 , is created. It consists of 4 assemblies with 50 subassemblies comprising 970 bodies, 24 materials and approximately 47000 outer subassembly faces. The 4 assemblies are the machine frame and the three sledges. The FE mesh has 1256497 nodes and 5094132 elements in total. The model itself is parametrized by about 400 functions (constants, lookup-tables, e.g.), 180 thermal boundary condition definitions and 109 thermal contacts between subassembly faces.

This results in an output-coupled 10 model with a total number of 287 inputs and 225 outputs for all subassemblies and an FE-coupled IO model with 69 inputs and 11 outputs.

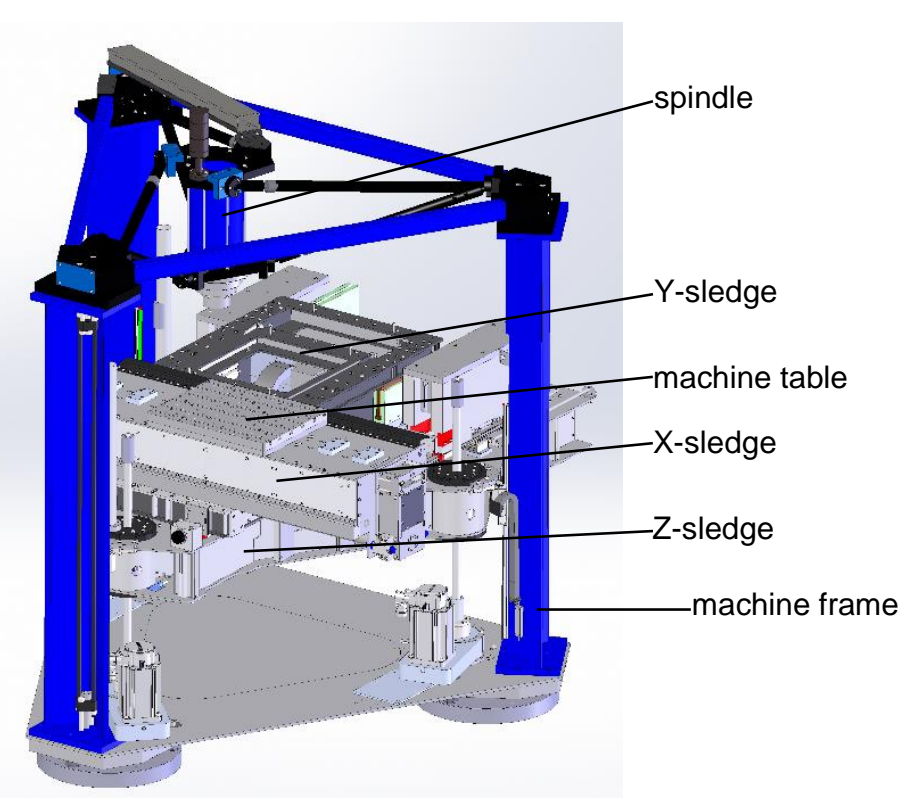

Fig. 2 : MAX CAD model. 


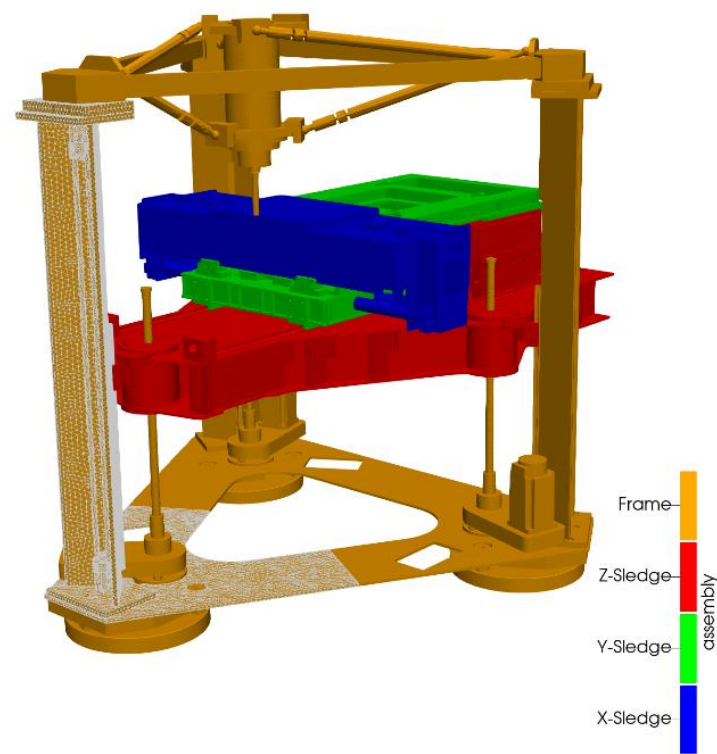

Fig. 3 : The four colored assemblies of the model. The bright white part of the column shows an excerpt of the mesh.

\subsection{Comparison of the ROMs with the Full-Order Model}

We applied the two MOR approaches for inhomogeneous initial conditions explained in Section 3.3 to the output-coupled as well as FE-coupled system of the thermal model of the demonstrator machine MAX. We used a constant initial temperature of $295.15 \mathrm{~K}$ for all nodes. The MOR method in use here for all model types, is Balanced Truncation. We computed reduced models with different truncation tolerances from $10^{-5}$ to $10^{-2}$ to provide an overview of the relation between reduced order and relative error in the outputs $\boldsymbol{y}$.

The full- and the reduced-order 10 systems were solved with the second-order Rosenbrock method from [Blom 1999] with a constant time step size. Due to the constant coefficient matrix $\boldsymbol{A}$, we reused the LU-decompositions over several time steps.

In the following, we examine the performance of the different reduced model types for one component $y_{1}$ of the outputs $\boldsymbol{y}$ by a comparison of the resulting temperature to that computed with the full models. Therefore, all models are driven by the same input function $\boldsymbol{u}(t)$. The corresponding output face is shown in Fig. 4.

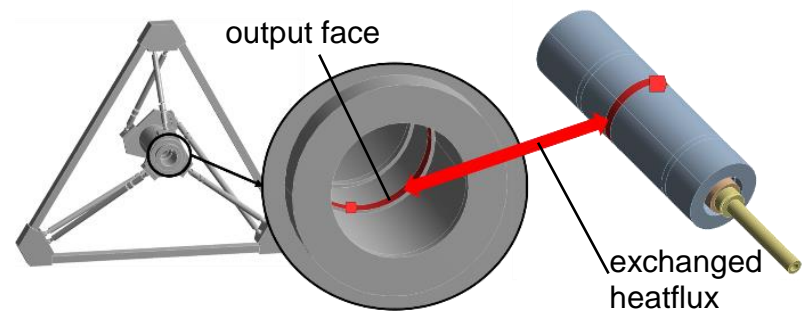

Fig. 4 : Detail view on subassembly contact investigated in output temperature comparison of $y_{1}$.

The relative error between the reduced and the full mode for the different truncation tolerances and reduction approaches is shown in Fig. 5 and 6, respectively. The associated temperature profile for a fixed truncation tolerance is examined in Fig. 7. In addition, Tab. 1 includes the corresponding maximal relative error for all outputs $y$, as well as the order of the ROMs. Notice that we chose the output $y_{1}$ which shows the largest error between ROM and full-order model for a detailed analysis. The error in the other output components is comparable or even smaller.

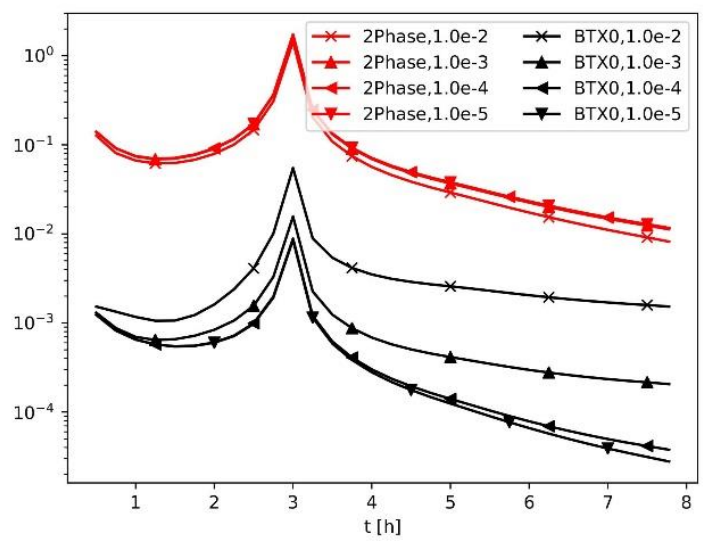

Fig. 5: Relative error between the reduced and the full output-coupled model in the first output $y_{1}$. Please see Fig. 7 for the temperature increase of the same output in time.

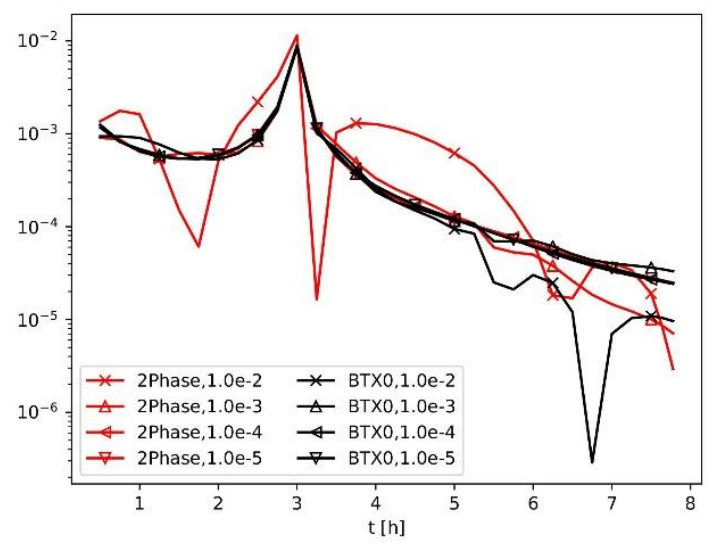

Fig. 6: Error between the FE-coupled full model and the FE-coupled reduced order model in the first output $y_{1}$.

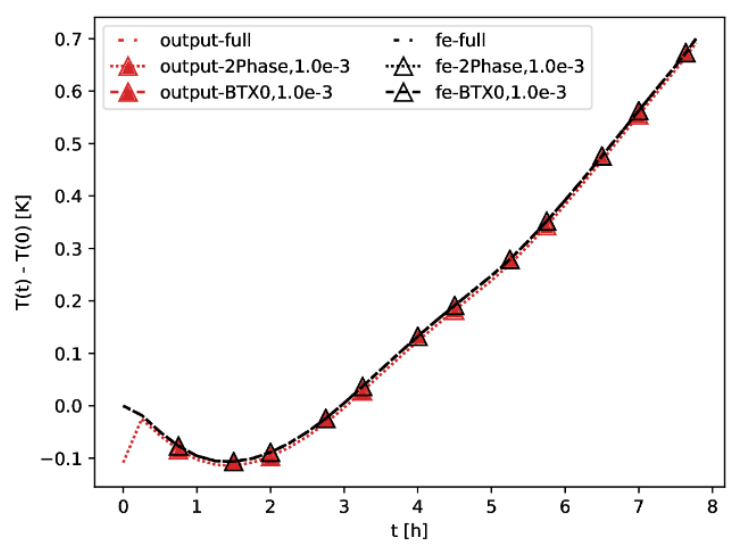

Fig. 7: Temperature increase with time step size 28.125 seconds for all combinations of both coupling strategies and initial value approaches, but one fixed truncation tolerance. 
While the depicted temperature increase over time with BTXO is visually indistinguishable from the full solution, we see a slight difference using the 2Phase approach. Please also note the large deviation at the very beginning of the simulation. This deviation stems from the initial value approximation with an impulse. Therefore, the initial phase in the reduced models was simulated with a much finer step size than in the transient phase. That error in the initial phase seems to dominate the whole simulation and overlays the truncation tolerance. But using even more steps in the initial phase would increase the simulation time even more and reduce the benefit from the order reduction.

Tab. 1 : Order of the reduced and the full models for both coupling strategies. Furthermore, we show the maximal relative error with respect to the solution of the full system.

\begin{tabular}{|c|c|c|c|c|c|}
\hline & tol & size & $\begin{array}{l}\text { 2Phase } \\
\text { max rel. err. }\end{array}$ & size & $\begin{array}{l}\text { BTXO } \\
\text { max rel. err. }\end{array}$ \\
\hline \multirow{4}{*}{ 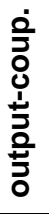 } & $1 e-2$ & 2308 & 1.44 & 1965 & 7.67e-02 \\
\hline & $1 e-3$ & 3005 & 1.70 & 2515 & $1.57 e-02$ \\
\hline & $1 e-4$ & 3707 & 1.73 & 3084 & 8.86e-03 \\
\hline & $1 e-5$ & 4399 & 1.73 & 3669 & $8.57 e-03$ \\
\hline \multirow{4}{*}{$\begin{array}{l}\text { 엉 } \\
\text { 을 } \\
\text { 언 } \\
\text { ய் }\end{array}$} & $1 e-2$ & 156 & 2.61e-02 & 138 & $1.18 e-02$ \\
\hline & $1 e-3$ & 196 & $9.01 e-03$ & 174 & $8.85 e-03$ \\
\hline & $1 e-4$ & 243 & 8.86e-03 & 216 & 8.80e-03 \\
\hline & $1 e-5$ & 295 & $8.68 e-03$ & 262 & 8.70e-03 \\
\hline
\end{tabular}

In the FE-coupled case the two approaches BTX0 and 2Phase show a comparable error behavior. Keep in mind, that the 2Phase system consists effectively of two reduced models, one with zero initial conditions and the inhomogeneous inputs (4) with $x_{0}=0$ and the second for the inhomogeneous initial conditions (12), which explains the differences in size. However, in the output-coupled case we observe a much better performance for the BTX0 approach.

Please note the drastic reduction in size for the FE-coupled approach. The storage needed for the system matrices of the $\mathrm{BTX} 0$ reduced FE-coupled model for tolerance $10^{-3}$ is just about $1 \mathrm{MB}$.

Furthermore, we wish to investigate the performance of the ROMs according to the simulation time while considering the effort of the reduction process. We show the total runtime of the time integration in Tab. 2 including the times for the application and decomposition of the Jacobian matrix. Interestingly, the solution of the full models is dominated by the applications of the decomposed Jacobian, whereas the reduced models are dominated by the decomposition itself. Furthermore, the strong coupling between the subassemblies forced us to integrate the systems of all subassemblies together in the output-coupled case.

Tab. 3 contains the necessary offline computation time for the generation of the various ROM types. Note that the different truncation tolerances have a negligible effect on the reduction time for $\mathrm{BT}$. We see that for the output-coupled case and using BTX0, the combined offline and simulation time is shorter than only one simulation of the full model. In the other cases, MOR gains when at least 3 to 4 model simulations are needed.

The runtimes for the reduced order models in combination with the FE-coupled model, shown in Tab. 2, are not a measurement error but emphasize the value of model order reduction.

Tab. 2: Total wall clock time for the simulation in seconds. The column tol represents the magnitude of the BT tolerance.

\begin{tabular}{|c|c|c|c|c|}
\hline & tol & full & 2Phase & BTXO \\
\hline \multirow{5}{*}{$\begin{array}{l}\text { 잉 } \\
\frac{0}{2} \\
\frac{0}{0} \\
\frac{0}{1} \\
\frac{1}{3} \\
\frac{2}{3} \\
0\end{array}$} & exact & 7279.39 & - & - \\
\hline & $1 e-2$ & - & 517.04 & 348.24 \\
\hline & $1 e-3$ & - & 946.13 & 619.57 \\
\hline & $1 e-4$ & - & 1573.69 & 1077.72 \\
\hline & $1 e-5$ & - & 2700.51 & 1693.94 \\
\hline \multirow{5}{*}{ 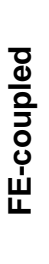 } & exact & 6600.72 & - & - \\
\hline & $1 e-2$ & - & 2.65 & 2.23 \\
\hline & $1 e-3$ & - & 3.91 & 3.12 \\
\hline & $1 e-4$ & - & 5.48 & 4.39 \\
\hline & $1 e-5$ & - & 7.44 & 6.06 \\
\hline
\end{tabular}

Tab. 3: Offline computation time in seconds of the ROMs for the different model types.

\begin{tabular}{|c|cc|}
\cline { 2 - 3 } \multicolumn{1}{c|}{} & 2Phase & BTX0 \\
\hline output-coupled & 7017 & 6464 \\
\hline FE-coupled & 23379 & 16804 \\
\hline
\end{tabular}

The observations in Section 3.2 in conjunction with the results given in Tab.1-3 clearly illustrate the importance of a conscious choice of the coupling strategy according to the intended use of the resulting ROM. It can be seen, that it is advisable to use a hybrid approach, which combines the benefits from both coupling approaches. Especially in the case of a relative movement between some parts of the model, output-coupling is recommended between these parts to avoid a parameter dependence in the system matrix $\boldsymbol{A}$, or the necessity for switching models. For a faster reduction and simulation process, it is recommended to consider the movement either in the input matrix $\boldsymbol{B}$ or the inputs $\boldsymbol{u}(t)$. For more information on the modeling and treatment of moving loads in the field of model order reduction see [Benner 2014]. An approach of BT for linear timevarying systems can be found in [Lang 2016].

On the other hand, FE-coupling is suggested to merge subassemblies with contact without relative movement to ensure a reasonable size of the reduced model.

\section{SUMMARY AND OUTLOOK}

The proposed methods along with the example simulation of a large, complex machine tool show the potential of the usage of MOR methods to reduce the simulation times and required storage capacity while preserving model accuracy. Especially the results for the reduced FE-coupled models are noteworthy.

The proposed workflow is not only applicable to machine tool models, but also appropriate for a wide range of thermal system simulations. Furthermore, the extension to more physical domains like elasticity is possible. Most importantly, the workflow allows the description of multi-physics problems. The description of thermo-elastic problems is already possible and published in [Beitelschmidt 2020]. 
The next steps include the incorporation of the elastic behavior into the simulation workflow to allow the online calculation of machine deformations.

The 2Phase method leads to an additional ODE for the inhomogeneous initial values. Our simulation results suggest that this block should be treated in a special manner. Particularly methods of higher order might be favorable in that framework.

\section{ACKNOWLEDGMENTS}

Funded by the German Research Foundation - Project-ID 174223256 - TRR 96

\section{REFERENCES}

[Antoulas 2005] Antoulas, A. C. Approximation of LargeScale Dynamical Systems. Philadelphia: SIAM Publications, 2005

[Antoulas 2008] Antoulas, A. C., et al. $\mathcal{H}_{2}$ model reduction for large-scale dynamical systems. SIAM J. Matrix Anal. Appl., 2008, Vol. 30, pp. 609-638

[Antoulas 2011] Antoulas, A. C., et al. Balanced truncation model reduction for systems with inhomogeneous initial conditions. Automatica, 2011, Vol. 47(3), pp. 559-564

[Bastian 2009] Bastian, P., et al. Infrastructure for the Coupling of Dune Grids. In: Numerical Mathematics and Advanced Applications, Uppsala, July 2009. Berlin, Heidelberg: Springer, pp. 107-114

[Baur 2014] Baur, U., et al. Model Order Reduction for Linear and Nonlinear Systems: A System-Theoretic Perspective. Archives of Computational Methods in Engineering, 2014, Vol. 21(4)

[Beattie 2017] Beattie, C., et al. Model Reduction for Systems with Inhomogeneous Initial Conditions. Systems \& Control Letters, 2017, Vol. 99, pp. 99-106

[Beitelschmidt 2020] Beitelschmidt, M., et. al. Simulation of the thermal behavior of machine tools for efficient machine development and online correction of the Tool Center Point (TCP)-displacement. In: Conference Proceedings on Thermal Issues, Aachen, 26-27 February, 2020. Bedford: euspen, pp. 135-138

[Benner 2006] Benner, P. Numerical Linear Algebra for Model Reduction in Control and Simulation. GAMMMitteilungen, 2006, Vol. 29(2), pp. 275 - 296

[Benner 2013] Benner, P. and Schneider, A. Balanced Truncation for Descriptor Systems with Many Terminals.
Preprint MPIMD/13-17. Max Planck Institute Magdeburg, 2013

[Benner 2014] Benner, P., et al. Model Order Reduction for Systems with Moving Loads. at - Automatisierungstechnik, 2014, Vol. 62(7), pp. 512-522

[Benner 2015a] Benner, P. and Feng, L. Model Order Reduction for Coupled Problems. Appl. Comput. Math., 2015, Vol. 14(1), pp.3-22

[Benner 2015b] Benner, P., et al. A Survey of ProjectionBased Model Reduction Methods for Parametric Dynamical Systems. SIAM Review, 2015, Vol. 57, No. 4, pp. 483-531

[Benner 2017] Benner, P., et al. Model Reduction and Approximation: Theory and Algorithms. Philadelphia: SIAM, 2017

[Benner 2020] Benner, P., et al. Matrix Equations, Sparse Solvers 2.0.1 -- Philosophy, Features and applications for (parametric) model order reduction. arXiv: 2003.02088, 2020

[Blom 1999] Blom, J. G., et al. A second-order Rosenbrock method applied to photochemical dispersion problems. SIAM Journal on Scientific Computing, 1999, Vol. 20, pp. $1456-1480$

[Hairer 2010] Hairer, E, Wanner, G. Solving Ordinary Differential Equations II. Berlin, Heidelberg: Springer-Verlag, 2010

[Hernandes-Becerro 2020] Hernandez-Becerro, P. Efficient Thermal Error Models of Machine Tools. PhD thesis. Zurich: ETH Zurich, 2020

[Herzog 2016] Herzog, R., et al. Parameteridentifikation in thermo-elastischen Systemen. In: Proceedings of the 4th Colloquium of SFB/Transregio 96, 2016, pp. 55-82 (in German)

[Ihlenfeldt 2019] Ihlenfeldt, S., et al. Online correction of thermal errors based on a structure model. International Journal of Mechatronics and Manufacturing Systems, 2019, Vol. 12(1), pp. 49 - 62

[Lang 2016] Lang, N., et al. Balanced truncation model reduction for linear time-varying systems. Mathematical and Computer Modeling of Dynamical Systems, 2016, Vol. 22(4), pp. 267-281

[Schroeder 2020] Schroeder, C. and Voigt, M. Balanced Truncation Model Reduction with A Priori Error Bounds for LTI Systems with Nonzero Initial Value. arXiv: 2006.02495, 2020 